Draft version SePtember 20, 2021

Preprint typeset using LTEX style emulateapj v. 04/17/13

\title{
MULTI-WAVELENGTH OBSERVATIONS OF 2HWC J1928+177: DARK ACCELERATOR OR NEW TEV GAMMA-RAY BINARY?
}

\author{
Kaya Mori ${ }^{1}$, Hongun An $^{2}$, Qi Feng ${ }^{3}$, Kelly Malone ${ }^{4}$, Raul R. Prado ${ }^{5}$, Yve E. Schutt ${ }^{1}$, Brenda L. Dingus ${ }^{4}$, E.V. Gotthelf ${ }^{1}$, \\ Charles J. Hailey ${ }^{1}$, Jeremy Hare ${ }^{6}$, Oleg Kargaltsev ${ }^{7}$, Reshmi MukherJee ${ }^{8}$ \\ Draft version September 20, 2021
}

\begin{abstract}
2HWC J1928+177 is a Galactic TeV gamma-ray sources detected by the High Altitude Water Cherenkov (HAWC) Observatory up to $\sim 56 \mathrm{TeV}$. The HAWC source, later confirmed by H.E.S.S., still remains unidentified as a dark accelerator since there is no apparent supernova remnant or pulsar wind nebula detected in the lower energy bands. The radio pulsar PSR J1928+1746, coinciding with the HAWC source position, has no X-ray counterpart. Our SED modeling shows that inverse Compton scattering in the putative pulsar wind nebula can account for the TeV emission only if the unseen nebula is extended beyond $r \sim 4^{\prime}$. Alternatively, $\mathrm{TeV}$ gamma rays may be produced by hadronic interactions between relativistic protons from an undetected supernova remnant associated with the radio pulsar and a nearby molecular cloud G52.9+0.1. NuSTAR and Chandra observations detected a variable X-ray point source within the HAWC error circle, potentially associated with a bright IR source. The X-ray spectra can be fitted with an absorbed power-law model with $N_{\mathrm{H}}=(9 \pm 3) \times 10^{22} \mathrm{~cm}^{-2}$ and $\Gamma_{X}=1.6 \pm 0.3$ and exhibit long-term X-ray flux variability over the last decade. If the X-ray source, possibly associated with the IR source (likely an O star), is the counterpart of the HAWC source, it may be a new TeV gamma-ray binary powered by collisions between the pulsar wind and stellar wind. Follow-up X-ray observations are warranted to search for diffuse X-ray emission and determine the nature of the HAWC source.
\end{abstract}

Keywords: gamma rays: ISM, - X-rays: general — pulsars: individual (PSR J1928+1746) — radiation mechanisms: non-thermal

\section{INTRODUCTION}

Over the last two decades, the advent of ground-based imaging air Cherenkov telescopes (IACTs) such as H.E.S.S., VERITAS and MAGIC uncovered a large number of TeV gamma-ray sources, most of which are associated with either pulsar wind nebulae (PWNe) or supernova remnants (SNRs). Identifying the nature of Galactic TeV gamma-ray sources is crucial for understanding the cosmic-ray acceleration mechanisms up to the $\mathrm{TeV}$ or PeV energy bands. Several TeV observations suggested the existence of the most extreme cosmic particle accelerator, the so-called Pevatron, in the Galactic Center (H.E.S.S. Collaboration et al. 2016) or elsewhere in our Galaxy (Xin et al. 2019). More recently, the High Altitude Water Cherenkov (HAWC) Observatory opened a new window for probing gamma-ray sources at even higher energies than the IACTs, $>100 \mathrm{TeV}$ (Abeysekara et al. 2017b, 2019). HAWC is a TeV gamma-ray telescope equipped with 300 water Cherenkov detectors (WCDs) which directly detects air shower particles produced by $\mathrm{TeV}$ gamma rays in the upper atmosphere and can collect data for sources continuously under all weather conditions. Therefore, the HAWC Observatory is more sensitive than IACTs at energies above $\sim 10 \mathrm{TeV}$ and uniquely explores astrophysical sources in the highest energy

\footnotetext{
kaya@astro.columbia.edu

${ }^{1}$ Columbia Astrophysics Laboratory, Columbia University, New York, NY 10027, USA

2 Department of Astronomy and Space Science, Chungbuk National University, Cheongju 28644, Republic of Korea

${ }^{3}$ Physics Department, Columbia University, New York, NY 10027, USA

${ }^{4}$ Los Alamos National Laboratory, Los Alamos, NM, USA

${ }^{5}$ DESY, Platanenallee 6, 15738 Zeuthen, Germany

${ }^{6}$ NASA Postdoctoral Program Fellow. NASA Goddard Space Flight Center, Greenbelt, MD 20771, USA

${ }^{7}$ Department of Physics, The George Washington University, 725 21st Street NW, Washington, DC 20052, USA

${ }^{8}$ Department of Physics and Astronomy, Barnard College, Columbia University, NY 10027, USA
}

gamma-ray band up to a few hundred TeV. About a half of the 39 Galactic HAWC sources have not been associated with previously known $\mathrm{TeV}$ sources detected by IACTs (Abeysekara et al. 2017a).

2HWC J1928+177 is one of the Galactic TeV sources detected by the HAWC standard point source search (Abeysekara et al. 2017a, Figure 1). Assuming a single powerlaw spectrum $\left(N(E) \propto E^{-\Gamma}\right)$ in the TeV band, the bestfit photon index is inferred to be $\Gamma=2.56 \pm 0.14$. The centroid of the source is $0.03^{\circ}$ away from the radio pulsar PSR J1928+1746 and $\sim 1.18^{\circ}$ away from another HAWC source, 2HWC J1930+188, which is associated with the SNR G54.1+0.3. The measured TeV flux in the whole region (Figure 1) for an extended source hypothesis is significantly larger than the sum of the point source fluxes for 2HWC J1928+177 and 2HWC J1930+188, which may imply that one or both of the sources are actually extended. H.E.S.S. later confirmed detection of 2HWC J1928+177 in the TeV band after improving the background subtraction method (Jardin-Blicq et al. 2019).

Taking into account the luminosity and spectral index of this source in the $\mathrm{TeV}$ band, the emission seems to match what is expected for a TeV PWN (H.E.S.S. Collaboration et al. 2018). The 83-kyr-old radio pulsar PSR J1928+1746 (Cordes et al. 2006) is a good counterpart candidate, as its position coincides well with the HAWC source position (Figure 1). However, the pulsar shows no PWN in the radio and X-ray bands despite its relatively high spin-down power $\left(\dot{E}=1.6 \times 10^{36} \mathrm{erg} \mathrm{s}^{-1}\right)$. There are two nearby Fermi sources in the 4FGL catalog (The Fermi-LAT collaboration 2019), but their positions do not overlap with the HAWC source; therefore, their association with the $\mathrm{TeV}$ emission is unlikely. For a SNR explanation to work, more than $10 \%$ of the SN energy would need to go towards the acceleration of $>1 \mathrm{TeV}$ protons, which is unlikely (Lopez-Coto et al. 2017). Given the lack of apparent environments for $\mathrm{TeV}$ photon production, 2HWC J1928+177 is possibly a unique $\mathrm{TeV}$ gamma-ray 
source whose origin is currently uncertain.

In this paper, we present multi-wavelength observations of 2 HWC J1928+177 and our investigation of the nature of the $\mathrm{TeV}$ emission. We first review recent gamma-ray observations of the HAWC source by VERITAS and H.E.S.S. (\$2) and by Fermi-LAT ( $\$ 3)$. We then describe the X-ray observations of the field made with $N u S T A R$ and Chandra and present the analysis results $(\$ 4)$. Then, in $\$ 5$, we discuss the nature of 2HWC J1928+177 using multi-wavelength spectral energy distribution (SED) data and models representing three possible scenarios: a pure leptonic case (PWN), a hadronic accelerator model (dark accelerator), and a TeV gamma-ray binary scenario. Finally, we summarize our results and future prospects in $\$ 6$.

\section{TEV GAMMA-RAY OBSERVATIONS OF 2HWC J1928+177 WITH VERITAS AND H.E.S.S}

VERITAS had previously observed the pulsar PSR J1928+1746 but only detected a 1.2- $\sigma$ excess at the pulsar location (Acciari et al. 2010). This discrepancy between the VERITAS and HAWC results seems to imply that there may be extended emission that is larger than VERITAS's point spread function (PSF). VERITAS recently published a study of 14 HAWC sources in the $2 \mathrm{HWC}$ catalog that are not associated with previously known $\mathrm{TeV}$ sources (Abeysekara et al. 2018). One of the regions discussed in detail was the part of the sky containing $2 \mathrm{HWC}$ $\mathrm{J} 1930+188$ and 2HWC J1928+177. 2HWC J1930+188 is associated with the $\mathrm{TeV}$ source previously identified by VERITAS, VER J1930+188 (Acciari et al. 2010), a known TeV PWN G54.1+0.3. Further analysis with VERITAS data found no emission from $2 \mathrm{HWC} \mathrm{J} 1928+177$ in either a point source (angular extension radius $<0.1^{\circ}$ ) or an extended source (angular extension radius $<0.23^{\circ}$ ) search. The flux upper limits $(99 \%$ confidence level) derived by VERITAS

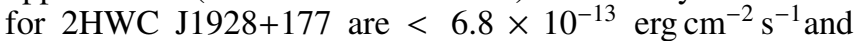
$<2.2 \times 10^{-12} \mathrm{erg} \mathrm{cm}^{-2} \mathrm{~s}^{-1}$, for the point and an extended source search, respectively. The total exposure time for this analysis is 44 hours (archival data analyzed for VERITAS ranges from 2007 to 2015) and the upper limits are calculated above an energy threshold of $460 \mathrm{GeV}$, assuming the photon index measured by HAWC $(\Gamma=2.56)$.

A H.E.S.S. study was recently carried out for comparing the Galactic plane as seen by HAWC and H.E.S.S. (JardinBlicq et al. 2019). Using a different background estimation for the H.E.S.S. analysis than employed previously, JardinBlicq et al. (2019) reported a detection of 2HWC J1928+177 as well as two other point sources above $5 \sigma$, each of which is less than half a degree away from the corresponding HAWC counterpart. It is interesting to note that this new technique seems promising for comparing IACT data with HAWC detections.

\section{Fermi-LAT OBSERVATIONS AND DATA ANALYSIS}

2HWC J1928+177 was not identified in the past FermiLAT catalogs. However, two sources within about $0.3^{\circ}$ from 2HWC J1928+177, 4FGL J1928.4+1801 and 4FGL $\mathrm{J} 1929.0+1729$, were identified in the 4FGL catalog, but not in the 3FHL catalog. We searched for a point source at the position of 2 HWC J1928+177 in Fermi-LAT data from the start of the mission to 2020 February. To avoid source confusion, we select photons between $1 \mathrm{GeV}$ and $2 \mathrm{TeV}$, as the PSF of LAT improves with energy and the $68 \%$ containment radius is below $1^{\circ}$ above $1 \mathrm{GeV}$. A power-law model leav-

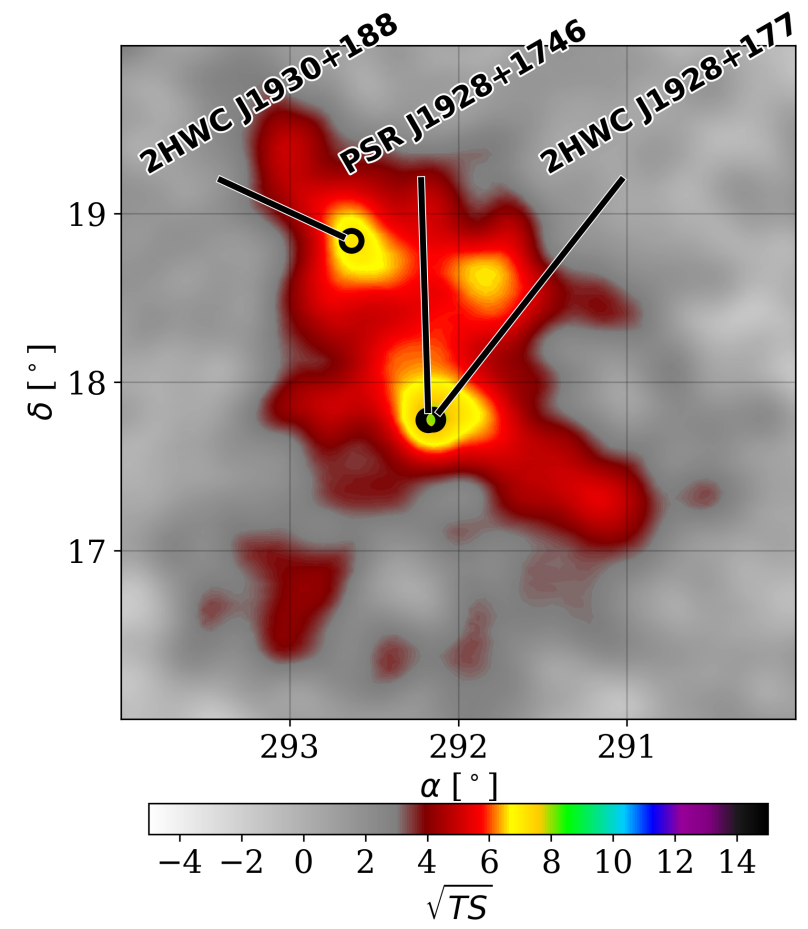

Figure 1. HAWC TeV skymap of the region around 2 HWC J1928+177 and $2 \mathrm{HWC} \mathrm{J} 1930+188$, both of which are modeled as point sources. The radio pulsar PSR J1928+1746 overlaps with the position of 2HWC J1928+177. The figure has been adapted from Abeysekara et al. (2017a).

ing photon index and normalization free at the position of $2 \mathrm{HWC}$ J1928+177, and all other sources in the 4FGL catalog were included in a likelihood analysis. No significant de-

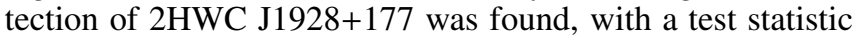
value of $4.1(2 \sigma)$, and a $95 \%$ upper limit on the energy flux

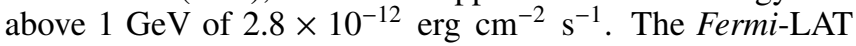
flux upper limits are used for multi-wavelength SED fitting in $\S 5$.

\section{X-RAY OBSERVATIONS AND DATA ANALYSIS}

We used a 90-ks NUSTAR observation (ObsID 30362002002) taken in 2017 June as a part of the NuSTAR-VERITAS-HAWC Legacy program which includes observations of PWN DA 495 (Coerver et al. 2019) and the TeV gamma-ray binary HESS J0632+057 (Archer et al. 2020), and two archival Chandra observations (ObsIDs 9081 and 22145) which were taken with $10 \mathrm{ks}$ exposures in 2008 and 2019, respectively. We also considered a handful of Swift/XRT observations of the field, but their short exposures yielded only a few counts in each observation, and so the Swift data are not very useful even after combining all the data.

\subsection{Data reduction}

The NUSTAR data were processed and analyzed using the NUSTARDAS v1.7.1 integrated in the HEASOFT 6.25 software package along with the NuSTAR Calibration Database (CALDB) files v20190513. The Chandra data were reprocessed with the chandra_repro tool of CIAO 4.11 to use the most recent calibration database.

\subsection{Image analysis}




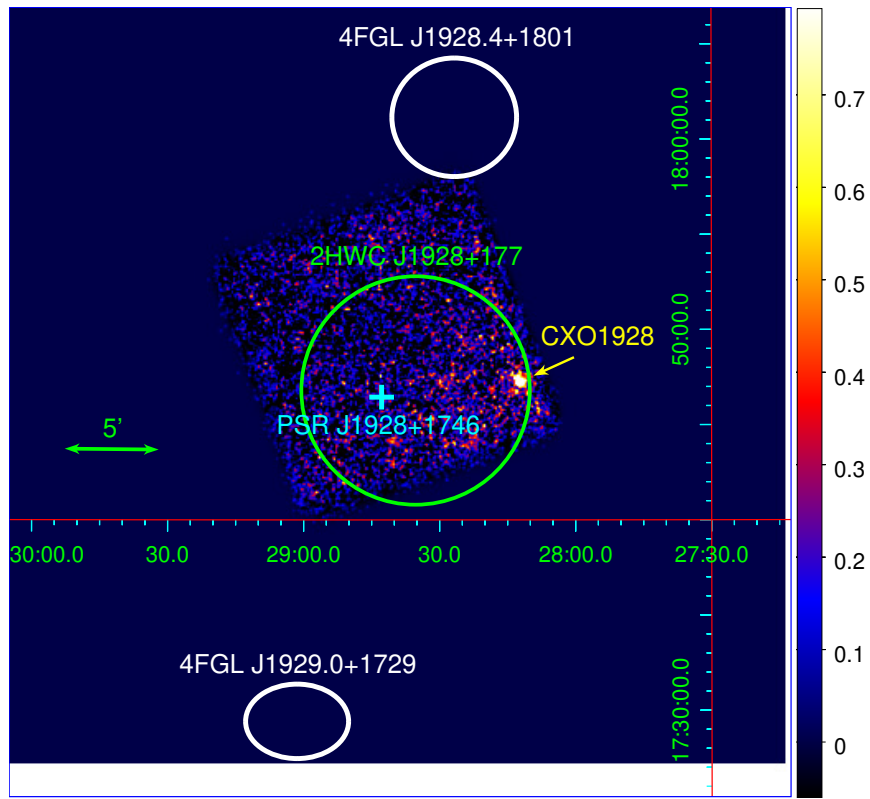

Figure 2. NUSTAR 3-20 keV background-subtracted image of 2HWC J1928+177 exhibiting the only X-ray source (CXO1928) in the NUSTAR FOV. The green circle indicates the position of the HAWC source and its $1 \sigma$ error. The positions of two Fermi sources with their $95 \%$ C.L. errors are shown as the white ellipses, while the cyan cross corresponds to the radio pulsar PSR J1928+1746.

We first obtained the NUSTAR background of the field for an image analysis. We used the nuskybgd software (Wik et al. 2014) to model the spatial and energy-dependent cosmic Xrays and a detector background. The background model components were determined by fitting the observed spectra in several source-free regions. The background spectra showed no significant $\mathrm{Fe}$ line at $E \sim 6-7 \mathrm{keV}$, indicating that the contamination from the Galactic ridge X-ray emission is negligible (Mori et al. 2015).

Figure 2 shows the background-subtracted NuSTAR image, after combining the two detector modules, in the 3 $20 \mathrm{keV}$ energy band. In the $13^{\prime} \times 13^{\prime}$ FOV, NUSTAR detected one hard X-ray source which coincides with a point source, CXO J192812.0+174712 (CXO1928 hereafter), originally detected by Chandra. CXO1928 is the brightest X-ray source detected in the hard X-ray band up to $\sim 20 \mathrm{keV}$ within the HAWC source position error circle. Using the CIAO tool wavdetect and the 2019 Chandra observation data, where CXO1928 was observed near the on-axis position, we determined the Chandra position to be RA $=19: 28: 12^{\prime \prime} .05$ and $\mathrm{DEC}=17: 47: 13^{\prime \prime} .35$ (J2000) with the $1-\sigma$ statistical error of $0.9^{\prime \prime}$.

We detected no X-ray source at the position of the radio pulsar, confirming the non-detection by Chandra. Using the $20 \mathrm{ksec}$ Chandra ACIS data, we determined the $2-8 \mathrm{keV}$ flux upper limits $(90 \%)$ of several circular regions around the radio pulsar position. For each source region, we computed the Poisson probability of detecting source counts over background counts (which were obtained from a region elsewhere on the ACIS FOV and corrected for different aperture sizes) and assumed a power-law spectrum with $\Gamma=2$ for calculating X-ray fluxes. We found that a $90 \%$ upper limit flux (unabsorbed) in the $2-8 \mathrm{keV}$ band is $7 \times 10^{-15}, 7 \times 10^{-14}$ and $3 \times 10^{-13} \mathrm{erg} \mathrm{cm}^{-2} \mathrm{~s}^{-1}$ for $r=3^{\prime \prime}, 1^{\prime}$ and $4^{\prime}$, respectively. These Chandra flux limits are useful for constraining diffuse
X-ray emission in both the leptonic and hadronic scenarios discussed in $\S 5.1$ and $§ 5.2$, respectively.

\subsection{Spectral and timing analysis}

We extracted NUSTAR spectra of CXO1928 from a $r=30^{\prime \prime}$ region and generated the $N u S T A R$ response matrix and ancillary response files using nuproducts. Background spectra were extracted from a $r=60^{\prime \prime}$ source-free region on the same detector chip. The net count rate in the 3-20 keV, after combining FPMA and FPMB spectra, is $0.003 \mathrm{cts} \mathrm{s}^{-1}$. The 2008 Chandra spectra (ObsID 9081) are extracted from a $r_{\text {major } / \text { minor }}=10 / 5^{\prime \prime}$ elliptical and a $r=10^{\prime \prime}$ circular region for the source (which is located at a large off-axis position) and background, respectively with the net source count rate of $0.012 \mathrm{cts} \mathrm{s}^{-1}$. The 2019 (ObsID 22145) Chandra spectra are extracted from a $r=2^{\prime \prime}$ circular region and $r=3-5^{\prime \prime}$ annular region for the source and background, respectively, yielding the $2-8 \mathrm{keV}$ net count rate of $0.005 \mathrm{cts} \mathrm{s}^{-1}$. The response files are generated with the specextract tool of CIAO 4.11. We grouped the spectra to ensure at least $30 \mathrm{cts} / \mathrm{bin}$ for $N u S T A R$ and $5 \mathrm{cts} /$ bin for Chandra, and jointly fit the spectra with an absorbed power-law model, employing the lstat statistic in XSPEC v12.10.1. Employing other statistics such as cstat or $\chi^{2}$ with gehrels weight does not alter the results significantly. We find that the absorbed power-law model with $\Gamma_{X}=1.6 \pm 0.3$ and $N_{\mathrm{H}}=(9 \pm 3) \times 10^{22} \mathrm{~cm}^{-2}$ describes the data well; the spectra are shown in Figure 3. However, we find that the source flux as measured by Chandra varies by a factor of 4-5 over 9 years. The large $N_{\mathrm{H}}$ implies a large distance to the source, and the $2-8 \mathrm{keV}$ luminosity is estimated to be $L_{X}=(0.6-1.9) \times 10^{33} \mathrm{erg} \mathrm{s}^{-1}$ in the low-flux state for an assumed distance of 5-10 kpc. Note that both NuSTAR and Chandra 2017-2019 observations yield X-ray (2-8 keV, absorbed) fluxes that are lower than the 2008 Chandra flux by a factor of $4-5$ (Figure 4 ) at the $\gtrsim 4 \sigma$ significance level. We also searched for an Fe line emission but did not find any significant emission.

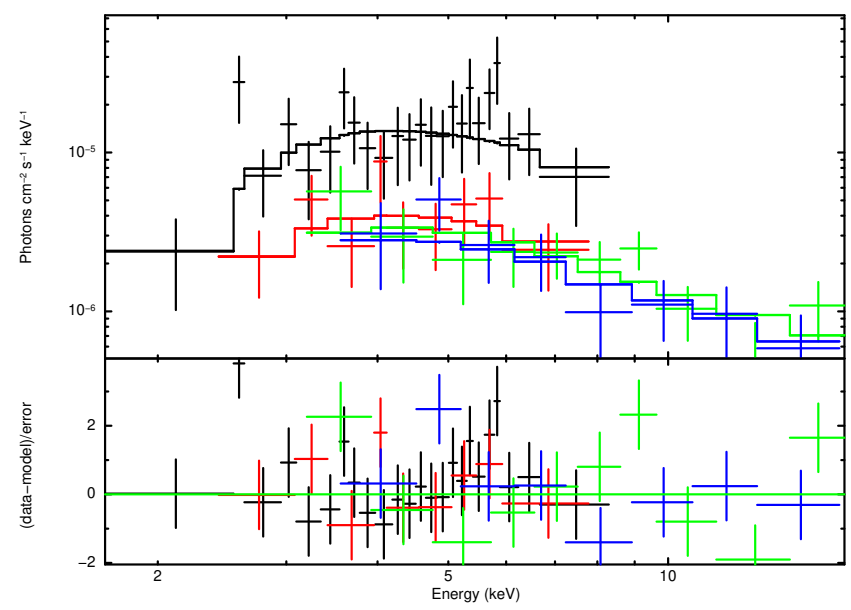

Figure 3. Chandra and NUSTAR spectra jointly fit with an absorbed powerlaw model. To account for X-ray flux variation, we fit a flux normalization factor between the Chandra ACIS (black: 2008, red: 2019) and the NuSTAR spectra (green: FPMA, blue: FPMB).

For NUSTAR timing analysis, we extracted source photon events within a $r=30^{\prime \prime}$ circle around the X-ray source. We then constructed 3-20 keV NuSTAR light curves and sub- 


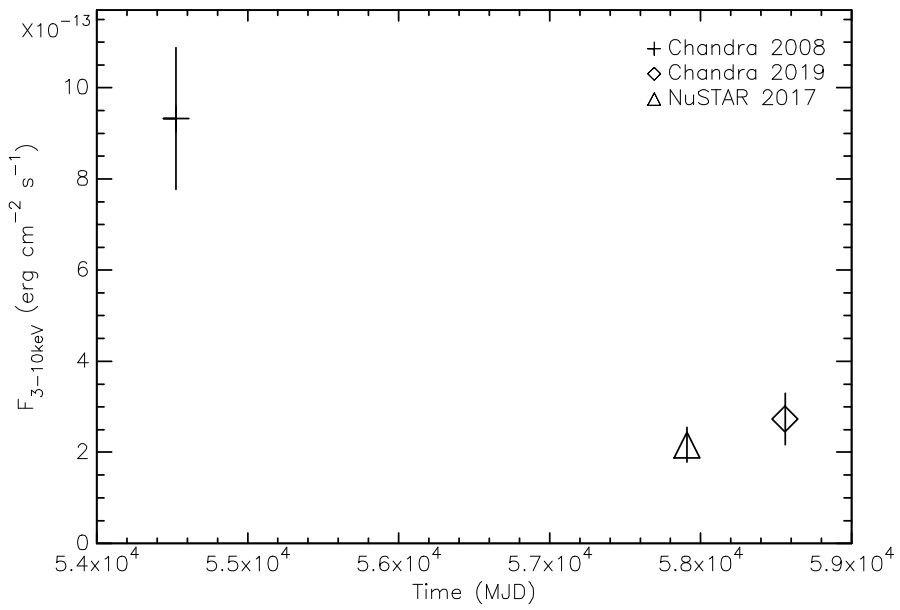

Figure 4. 3-10 keV lightcurve of CXO1928 over the last decade from two Chandra observations (in 2008 and 2019) and one NuSTAR observation (in 2017). The source was brighter in 2008 than in 2017-2019 by a factor of $\sim 4$ HESS and VERITAS observations were performed in 2015-2017, when the source was likely dimmer.

tracted background light curves after the proper normalization. We found no significant modulation in the NuSTAR lightcurves. Furthermore, we found no evidence of aperiodic variability (i.e. red noise) in the power density spectra produced from the NUSTAR data. The 3-20 keV power density spectra are consistent with a flat white noise component, unlike accreting X-ray pulsars which often shows strong red noise components (Lazzati \& Stella 1997).

\subsection{A potential IR counterpart of CXO1928}

We searched IR-to-optical catalogs for a counterpart of the X-ray source CXO1928. There is a bright IR source (2MASS J19281204+1747111) with magnitudes of $J=17.8$, $H=14.7$ and $K=13.6$, offset by $2.2^{\prime \prime}$ from the Chandra position of CXO1928. Although the offset is large compared to the statistical error of the Chandra position of $0.9^{\prime \prime}(1-\sigma)$, the systematic errors can be as large as $\sim 1.4^{\prime \prime}\left(99 \%\right.$ CL) ${ }^{9}$. Unfortunately, there is no X-ray source in the Chandra FOV that can be registered to its known IR or radio position for a boresight correction. Therefore, the association cannot be firmly established with the source positions. Alternatively, based on the surface density of nearby IR sources (which are brighter than $K=13.6$ ) in the 2MASS catalog, we estimated that the probability of chance coincidence between the Chandra and IR sources is 3\%; their association is only suggestive. Assuming an optical extinction $A_{V}>17$, as indicated by the large $N_{\mathrm{H}}$ measured from $\mathrm{X}$-ray spectral fitting and using the relation in Güver \& Özel (2009), we fit a stellar SED model to the existing IR flux data from Spitzer, UKIRT, and 2MASS which suggests the IR source is a massive star (likely O-type).

\section{DISCUSSION}

In this section, we consider three scenarios for the $\mathrm{TeV}$ emission from 2HWC J1928+177: (1) a putative PWN of the radio pulsar PSR J1928+1746, (2) hadronic interactions in the unseen SNR shock and (3) a TeV gamma-ray binary (TGB). Our investigation is largely based on the multi-wavelength SED including Chandra, NuSTAR, Fermi and HAWC data. In the first two cases, we assume that the variable X-ray

${ }^{9}$ https://cxc.harvard.edu/cal/ASPECT/celmon/ source CXO1928 is not associated with the HAWC source, and therefore the X-ray SED is unconstrained. In the TGB case, we assume that both the X-ray source and bright IR counterpart candidate are associated with the HAWC source. We do not consider an extra-galactic origin, such as hardTeV blazars (MAGIC Collaboration et al. 2019) because the scenario seems implausible due to the lack of a radio galaxy counterpart (van Velzen et al. 2012) as well as no short-term $(\sim$ hours) variability from the X-ray source (Pandey et al. 2017).

\subsection{A putative PWN of the radio pulsar PSR J1928+1746}

PSR J1928+1746 is one of the leading counterpart candidates for the HAWC source due to its positional coincidence. However, no nebula has been detected around the pulsar in the radio band (Cordes et al. 2006). Chandra observations yielded no X-ray detection of the pulsar, setting an upper limit of the unabsorbed flux in the $2-8 \mathrm{keV}$ band $F_{\mathrm{X}}<7 \times 10^{-15} \mathrm{erg} \mathrm{cm}^{-2} \mathrm{~s}^{-1}$ and $<3 \times 10^{-13} \mathrm{erg} \mathrm{cm}^{-2} \mathrm{~s}^{-1}$, assuming that the putative PWN is extended over $r=3^{\prime \prime}$ and $r=4^{\prime}$, respectively.

In PWN models, it is believed that synchrotron radiation produces radio to X-ray photons, and inverse Compton upscattering of the synchrotron (self-Compton; SSC) and/or the external IR/CMB radiation fields produces the $\mathrm{TeV}$ emission. In order to bound some of the PWN parameters, we applied the leptonic model, InverseCompton+Synchrotron, in the naima software package (Zabalza 2015) to the multiwavelength SED data. If we assume a compact PWN $\left(r \lesssim 1^{\prime}\right)$, the very-high TeV-to-X-ray flux ratio of $2 \mathrm{HWC} \mathrm{J} 1928+177$ $\left(F_{\mathrm{TeV}} / F_{\mathrm{X}} \gtrsim 100\right)$ requires the PWN B-field far below the typical ISM value $\left(B_{\mathrm{PWN}} \lesssim 1 \mu \mathrm{G}\right.$ whereas $B_{\mathrm{ISM}} \sim 10 \mu \mathrm{G}$; Crutcher 2012) and/or extremely high NIR and FIR densities at $U_{\gamma} \sim 10^{3}-10^{4} \mathrm{eV} \mathrm{cm}^{-3}$ for the typical PWN Bfield range $(B \sim 10-100 \mu \mathrm{G}$; Martin et al. 2014). The latter case is implausible since such a high radiation density in the IR/optical band is only observed in the Galactic Center (Davidson et al. 1992). Alternatively, the PWN radius can be reduced to $R_{\mathrm{PWN}} \sim 10^{-5} \mathrm{pc}$, as a result of SNR reverse shocks crushing the nebula (Reynolds \& Chevalier 1984), in order to amplify the SSC component to fit the TeV spectra. However, the shock compression amplifies the magnetic field strength, and thus the synchrotron emission should be detectable in the radio and X-ray bands (Gelfand et al. 2007).

We found that the pure leptonic case is marginally plausible only when we assume a large PWN size of $r \sim 4^{\prime}$ or $r \sim 6 \mathrm{pc}$ at the pulsar distance of $5.8 \mathrm{kpc}$ (Nice et al. 2013), thus relaxing the X-ray flux upper limit to $<3 \times 10^{-13} \mathrm{erg} \mathrm{cm}^{-2} \mathrm{~s}^{-1}$. According to Bamba et al. (2010) who studied the X-ray PWN size variation with the spin-down age, the 83-kyr old pulsar may well be extended beyond $r \sim 6 \mathrm{pc}$. For example, as shown in Figure 5, the SED data from X-ray to TeV bands can be fit with $B_{\text {PWN }} \sim 5 \mu \mathrm{G}$ and elevated IR radiation densities $\left(U_{\mathrm{NIR}}=1 \mathrm{eV} \mathrm{cm}^{-3}\right.$ and $\left.U_{\mathrm{FIR}}=10 \mathrm{eV} \mathrm{cm}^{-3}\right)$. Bamba et al. (2010) argued that such a extended, faint PWN can have its magnetic field strength decayed to below the typical ISM $B$-field. Also, more recent observations of the region using WISE and Gaia data found five star clusters (Cmg 495, Cmg 497, Cmg 498, Cmg 499, and Liu \& Pang catalog ID 1262) within $r \sim 8^{\prime}$ from the pulsar position, some of which could be embedded in molecular clouds (Camargo et al. 2015; Liu \& Pang 2019). These star clusters can contribute to enhancing ICS emission to the TeV flux level observed by HAWC. 


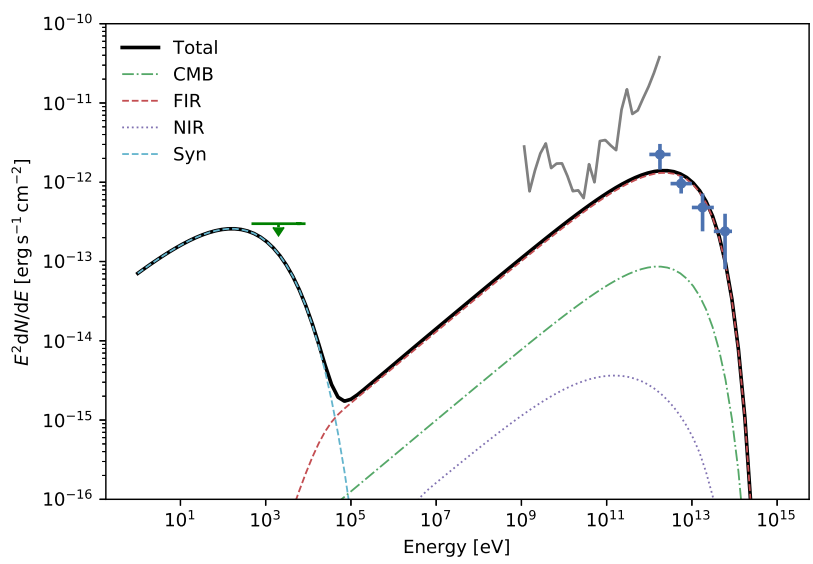

Figure 5. A SED plot for the PWN scenario with $R_{\mathrm{PWN}}=6 \mathrm{pc}$. The FermiLAT flux upper limits and HAWC TeV flux data are plotted as the grey line and blue points, respectively. The X-ray flux upper limits (green arrow) were determined from a $r=4^{\prime}$ circle around the radio pulsar position using the Chandra ACIS data. The radiation densities in the NIR and FIR bands are set to the values of $1 \mathrm{eV} \mathrm{cm}^{-3}$ and $10 \mathrm{eV} \mathrm{cm}^{-3}$. We adopted a cutoff power-law model for the electron energy spectrum with $\alpha_{e}=2.1$ and $E_{\mathrm{cut}}=30 \mathrm{TeV}$ and a PWN magnetic field strength of $B=5 \mu \mathrm{G}$ as a representative case.

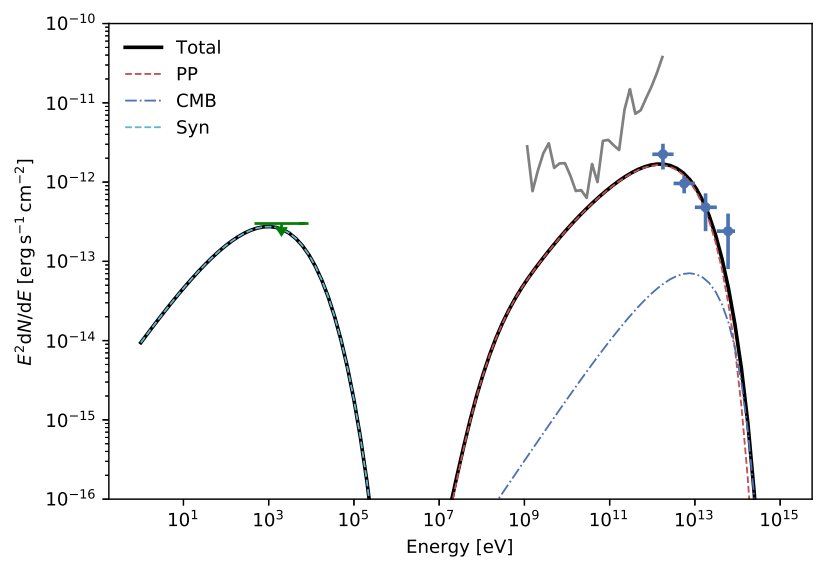

Figure 6. A SED plot in the hadronic scenario, using the hadronic PionDecay and leptonic models in the naima package. The Fermi-LAT flux upper limits and HAWC TeV flux data are plotted as the grey line and blue points, respectively. For the leptonic model, we assumed only the CMB as a source of seed photons for the ICS component and a magnetic field strength of $B=5 \mu \mathrm{G}$. The X-ray flux upper limits (green arrow) were determined from a $r=4^{\prime}$ circle around the radio pulsar position using the Chandra ACIS data. Parameters are $\alpha_{p}=1.4, E_{\text {cut }}=40 \mathrm{TeV}$ and $n_{\mathrm{H}}=130 \mathrm{~cm}^{-3}$ (i.e., the mean hydrogen density of the molecular cloud G52.9+0.1). We adopted the same parameters for the electron and proton energy spectra except for the normalization factors. The total energy of the relativistic protons is $W_{\mathrm{p}}=4.8 \times 10^{47}$ erg $(1-10 \mathrm{TeV})$ or $2.1 \times 10^{48} \mathrm{erg}(0.01-100 \mathrm{TeV})$. The total energy of the relativistic electrons should be lower than $W_{\mathrm{e}}=7.1 \times 10^{45} \mathrm{erg}(1-10 \mathrm{TeV})$ or $2.1 \times 10^{46}$ erg $(0.01-100 \mathrm{TeV})$.

Therefore, we conclude that a diluted, unseen PWN associated with the radio pulsar can account for the TeV emission. A deeper X-ray survey around the pulsar may uncover diffuse X-ray emission like other faint X-ray PWNe detected by Bamba et al. (2010).

\subsection{Hadronic interactions}

Alternatively, the $\mathrm{TeV}$ emission could originate primarily from hadronic interactions as a result of collisions between relativistic protons and the ISM or nearby molecular clouds.
Pion decays from proton-proton collisions are efficient $\mathrm{TeV}$ emitters, whereas the ICS component from $\gtrsim 100-\mathrm{TeV}$ electrons is suppressed at $E_{\gamma} \gtrsim 10 \mathrm{TeV}$ due to the Klein-Nishina effect (Rieger et al. 2013). The molecular cloud G52.98+0.18 in the HAWC source region can serve as a target for hadronic interactions (Rice et al. 2016). From the molecular cloud's measured mass, angular size, and distance of $4.49 \times 10^{5} \mathrm{M}_{\odot}$, $0.22^{\circ}$, and $9.56 \mathrm{kpc}$, respectively, we estimated that the average hydrogen density is $130 \mathrm{~cm}^{-3}$. There is no radio or X-ray SNR within $r \sim 30^{\prime}$ from the HAWC source; however, soft X-ray emission from the putative SNR may be strongly absorbed. We assumed a putative SNR with the shell radius derived from the Sedov solution of $r_{s} \propto\left(E t_{0}^{2} / n_{0}\right)^{1 / 5}$ where $E=10^{51}$ erg (the total SN energy released), $t_{0}=83 \mathrm{kyrs}$ (the spin-down age of the radio pulsar) and $n_{0}$ (the mean number density of the molecular cloud). We estimated that the SNR shell radius should be $\sim 11 \mathrm{pc}$ or $\sim 4^{\prime}$ assuming that the source is located at the distance of the molecular cloud at $10 \mathrm{kpc}$ (Rice et al. 2016). Alternatively, if we adopt the distance to the pulsar (5.8 kpc; Nice et al. 2013), the angular radius of the SNR should be $\sim 7^{\prime}$.

To explore the hadronic scenario, we applied a combination of the leptonic and hadronic models in the naima package to the multi-wavelength SED data. We assumed that the particle energy spectrum follows a power-law with an exponential cutoff $\left(N(E) \propto E^{-\alpha} e^{-E / E_{\text {cut }}}\right)$ because a single powerlaw model does not fit the SED. First, we fit the PionDecay model only in the gamma-ray band. A hard proton spectral index $\left(\alpha_{p}=1.4\right)$ and an exponential cutoff at $E_{\text {cut }}=40 \mathrm{TeV}$ are required to give a SED model consistent with the Fermi $\mathrm{GeV}$ upper limit and HAWC TeV spectra. The total energy of relativistic protons $(0.01-100 \mathrm{TeV})$ is $W_{p}=2.1 \times 10^{48} \mathrm{erg}$. This corresponds to a small fraction $(<0.1 \%)$ of the typical supernova energy $\left(\sim 10^{51} \mathrm{erg}\right)$.

Since the naima package does not track the by-product leptons from the PionDecay model, we added a separate leptonic model to constrain the secondary electron population so that their synchrotron emission does not over-predict the radio and X-ray flux upper limits. Note that the ICS component from the electron population needs to have a small contribution so as to not overshoot the TeV fluxes. Assuming the same energy spectrum for electrons and protons (i.e., $N(E) \propto E^{-\alpha} e^{-E / E_{\text {cut }}}$ with $\alpha=1.4$ and $E_{\text {cut }}=40 \mathrm{TeV}$ ) and a typical ISM magnetic field strength $\left(B_{\mathrm{ISM}}=5 \mu \mathrm{G}\right.$; Crutcher 2012), we found that the total energy of relativistic electrons $\left(N_{e}\right)$ should be less than $W_{e}=2.1 \times 10^{46} \mathrm{erg}(0.01-100 \mathrm{TeV})$ so as not to exceed the X-ray flux upper limit from a $r=4^{\prime}$ 'region around the radio pulsar (Figure 6). If the putative SNR is located at the molecular cloud distance $(\sim 10 \mathrm{kpc})$, its angular size is estimated to $r \sim 7^{\prime}$, which is larger than the FOV of the Chandra ACIS observations. Assuming that diffuse X-ray emission associated with the putative SNR is spatially uniform, the X-ray flux upper limit is higher by a factor of $\sim 3$ thus enhances the electron energy to $W_{e}=6.3 \times 10^{46} \mathrm{erg}$. However, the small ratio of $W_{e} / W_{p} \sim 0.01-0.03$ is still difficult to reconcile with the $\mathrm{p}$-p collision case since the total kinetic energy of secondary electrons (i.e. byproducts of charged pion decays) should be $\sim 1 / 3$ of the total radiation energy of $\pi^{0}$ gamma-rays (Coerver et al. 2019). In order to yield the ratio $W_{e} / W_{p}$ comparable to $\sim 1 / 3$, it requires a lower ambient $B$-field, which seems implausible within a molecular cloud where the B-field should be amplified. Only if the extent of relativistic proton population is larger than $r \sim 7^{\prime}$ (e.g., the SNR may be older than 
the spin-down age), the resultant synchrotron emission may be consistent with the X-ray flux limits while $B_{\mathrm{ISM}} \sim 5 \mu \mathrm{G}$.

It is therefore possible that relativistic protons in a diluted, undetected SNR, extending over a $r \gtrsim 4^{\prime}$ region, produce $\mathrm{TeV}$ gamma-rays via collisions with the molecular clouds. Thus, diffuse X-ray or radio emission associated with the HAWC source could be too faint to be detected (Butt et al. 2008). Prior to observations by the Cherenkov Telescope Array (CTA) which may resolve the $\mathrm{TeV}$ emission (Cherenkov Telescope Array Consortium et al. 2019), it is essential to survey a larger region around the HAWC source with X-ray telescopes. Some TeV sources are associated with extended diffuse X-ray sources, and even non-detection of an X-ray counterpart can be useful for inferring the source type. XMMNewton, with its large FOV, is best suited to search for other $\mathrm{X}$-ray counterpart candidates in a larger region around the HAWC source.

\subsection{A new TeV gamma-ray binary?}

If the variable X-ray source CXO1928 is associated with the HAWC source, it may belong to a rare class of $\mathrm{TeV}$ gamma-ray binaries (Dubus 2013). These systems are likely composed of a neutron star (NS) orbiting around a massive $\mathrm{O} / \mathrm{B}$ star. The exact mechanism responsible for the nonthermal emission is still unknown. However, a possible scenario is that $e^{ \pm}$pairs from the pulsar wind are accelerated at the shock produced by the interaction between the pulsar and the stellar wind. The resultant high-energy electron population emits synchrotron and ICS radiation which accounts for the observed X-ray and gamma-ray emission, respectively (Tavani \& Arons 1997).

The X-ray spectral and timing signatures of CXO1928 a single power-law spectrum with $\Gamma_{X} \sim 1.6$, long-term time variability and X-ray luminosity - are consistent with those of other TGBs with massive (O or B) companion stars (Dubus 2013); such variability can be explained as due to varying $B$ or bulk Doppler boost as the system geometry changes with orbital phases (e.g., An \& Romani 2017). Unlike accreting $\mathrm{X}$-ray binaries, the lack of X-ray aperiodic variability shorter than a day is consistent with the TGB scenario (Mori et al. 2017). In addition, both the $\mathrm{TeV}$ gamma-ray photon index $\left(\Gamma_{\mathrm{TeV}}=2.6\right)$ and the TeV to $\mathrm{X}$-ray flux ratio $\left(F_{\mathrm{TeV}} / F_{X}=0.4-\right.$ 2) are in the typical parameter range for other $\mathrm{TeV}$ gamma-ray binaries (Dubus 2013).

In order to further probe the TGB hypothesis, we compared the NUSTAR and HAWC SED data to a generic, analytical model based on the NS assumption (see more details in Archer et al. 2020). In this model the energy spectrum of the high-energy electron population is described by a power law with an exponential cutoff. While the normalization and the slope of the power law were obtained by fitting the $N u S$ $T A R$ data, the cutoff energy $\left(E_{\text {cut }}\right)$ was set to $100 \mathrm{TeV}$, which is assumed to be a minimum value to describe the HAWC observations. The typical $B$-field strength within this scenario is 0.01-1 G (Archer et al. 2020). In Figure 7, we show the SED comparison in two cases in which $B=0.01 \mathrm{G}$ (top) and $B=0.1 \mathrm{G}$ (bottom). For values higher than that, it was found that the expected flux in the $\mathrm{GeV}$ band is too large and could not accommodate the lack of detection by Fermi-LAT.

From the high-energy electron spectrum obtained by fitting the NUSTAR data, we calculated the expected flux of gamma rays produced by ICS. The ICS photon field in this scenario is given by thermal UV photons from the O/B companion star, which we assumed to be at the typical temperature of $3 \times 10^{4}$
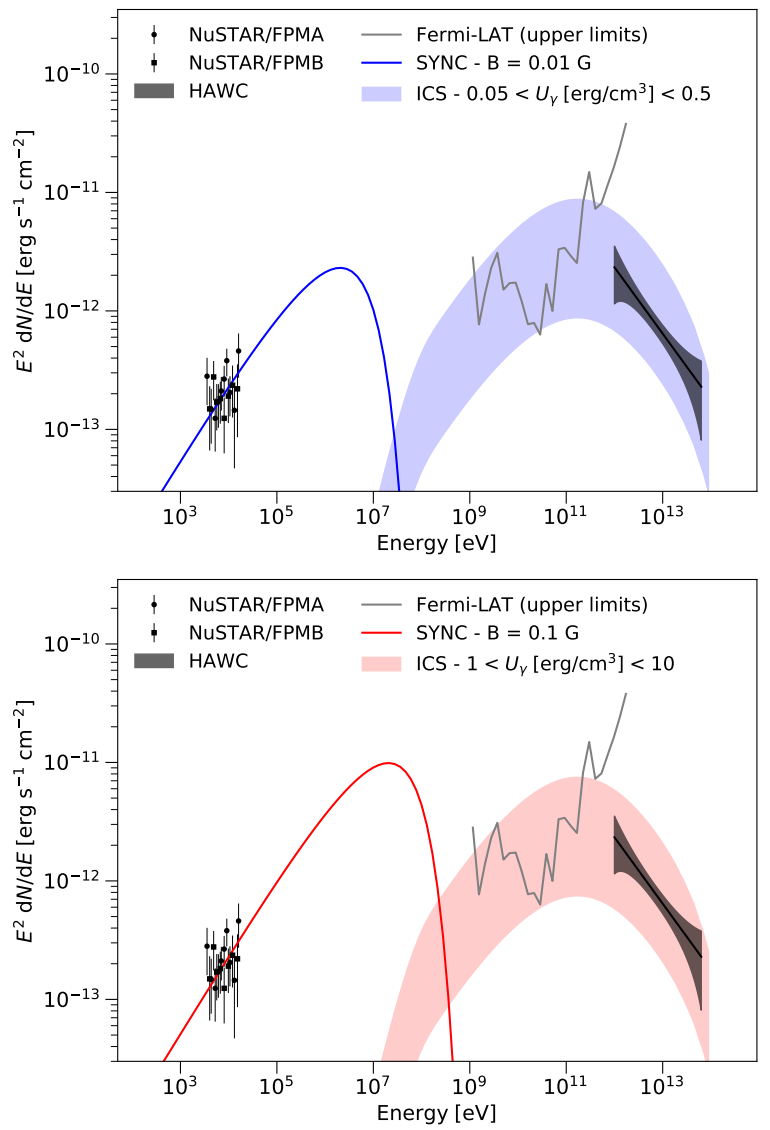

Figure 7. A TGB model fit to the X-ray and gamma-ray SED data with $B=$ $0.01 \mathrm{G}$ (top panel) and $B=0.1 \mathrm{G}$ (bottom panel). The Fermi/LAT 5- $\sigma$ upper limits are indicated by dotted lines. The colored lines and bands represent the synchrotron and ICS components of the TGB SED model, respectively.

$\mathrm{K}$. The photon density $\left(U_{\gamma}\right)$ varies substantially with the distance of the shock from the companion star and therefore is strongly dependent on the geometry of the system, which is unknown.Besides that, $\gamma-\gamma$ absorption within the system, which is also geometry dependent, may also affect the observed gamma-ray flux. Due to these limitations, it is only possible to loosely constrain the SED at the gamma-ray band. In Figure 7, we show filled bands for the ICS spectra for a given range of $U_{\gamma}$ that are reasonably expected for TGBs and would also be consistent with the HAWC data. Although the NuSTAR and the HAWC data were taken at different epochs and TGBs are very variable sources, the SED comparison shows that the data are consistent with the expectation from a TGB scenario. However, further broadband studies are required to confirm the TGB scenario, and particularly, detection of $\mathrm{TeV}$ variability would be a smoking gun. If confirmed, 2HWC J1928+177 may be a unique binary system emitting gamma-rays up to $\sim 100 \mathrm{TeV}$ since no other known TGBs have been detected by HAWC above $E \sim 10 \mathrm{TeV}$ (Rho 2017).

\section{SUMMARY}

- 2HWC J1928+177 is one of the Galactic TeV sources detected by HAWC up to $\sim 56 \mathrm{TeV}$ and later confirmed by H.E.S.S. It is still unclear if the TeV source is extended or not. There is no SNR, PWN or Fermi source coinciding with the HAWC source position. 
- The 83-kyr-old radio pulsar PSR J1928+1746 can account for the $\mathrm{TeV}$ emission in the pure leptonic scenario, only if its putative PWN is extended beyond $r \sim 4^{\prime}$. An alternative scenario could involve an unseen SNR whose shock produces the $\mathrm{TeV}$ emission via relativistic protons colliding with a nearby molecular cloud. The estimated proton energy $\left(W_{p}=4.7 \times 10^{47} \mathrm{erg}\right)$ is reasonable as only a small fraction $(\sim 0.1 \%)$ of the supernova energy is required to power the $\mathrm{TeV}$ emission. In the hadronic scenario, diffuse X-ray emission from secondary electron synchrotron radiation over $r \gtrsim 7^{\prime}$ should be present in the region.

- NUSTAR and Chandra detected a bright X-ray source CXO1928 which overlaps with the HAWC source position. The non-thermal X-ray spectra, long-term X-ray flux variation, the lack of aperiodic variability on a time scale shorter than a day and a potential association with a bright IR source suggests the HAWC source may be a new $\mathrm{TeV}$ gamma-ray binary. However, it needs to be confirmed by detecting variability in the $\mathrm{TeV}$ band. If the $\mathrm{TeV}$ source turns out to be extended, this scenario may be less likely.

- The HAWC source, given its location in a complex region with star clusters and molecular clouds, may be composed of several $\mathrm{TeV}$ sources such as a faint (undetected) nebula of the radio pulsar and diffuse $\mathrm{TeV}$ emission from hadronic interactions and may be spatially resolved by the near-future CTA observatory. Until then, a large/deep X-ray survey around the HAWC source may provide clues of the source type. XMM-Newton, given its large FOV, is best suited to search for other Xray counterpart candidates in a larger region around the HAWC source.

This work made use of data from the NUSTAR mission, a project led by the California Institute of Technology, managed by the Jet Propulsion Laboratory, and funded by the National Aeronautics and Space Administration. We thank the NUSTAR Operations, Software and Calibration teams for support with the execution and analysis of these observations. This research has made use of the NUSTAR Data Analysis Software (NuSTARDAS) jointly developed by the ASI Science Data Center (ASDC, Italy) and the California Institute of Technology (USA). This research is supported by grants from the U.S. Department of Energy Office of Science, the U.S. National Science Foundation and the Smithsonian Institution, and by NSERC in Canada. We acknowledge the excellent work of the technical support staff at the Fred Lawrence Whipple Observatory and at the collaborating institutions in the construction and operation of the VERITAS instrument. H.A. acknowledges support from Basic Science
Research Program through the National Research Foundation of Korea (NRF) funded by the Ministry of Science, ICT \& Future Planning (NRF-2017R1C1B2004566). J.H. acknowledges support from an appointment to the NASA Postdoctoral Program at the Goddard Space Flight Center, administered by the USRA through a contract with NASA. O.K. was supported by the National Aeronautics and Space Administration through the award 80NSSC19K0576.

\section{REFERENCES}

Abeysekara, A. U., Albert, A., Alfaro, R., et al. 2017a, ApJ, 843, 40 -. 2017b, ApJ, 843, 39

Abeysekara, A. U., Archer, A., Benbow, W., et al. 2018, ApJ, 866, 24 Abeysekara, A. U., Albert, A., Alfaro, R., et al. 2019, ApJ, 881, 134

Acciari, V. A., Aliu, E., Arlen, T., et al. 2010, ApJ, 719, L69

An, H., \& Romani, R. W. 2017, ApJ, 838, 145

Archer, A., Benbow, W., Bird, R., et al. 2020, ApJ, 888, 115

Bamba, A., Anada, T., Dotani, T., et al. 2010, ApJ, 719, L116

Butt, Y. M., Combi, J. A., Drake, J., et al. 2008, MNRAS, 385, 1764

Camargo, D., Bica, E., \& Bonatto, C. 2015, Monthly Notices of the Royal Astronomical Society, 455, 3126

Cherenkov Telescope Array Consortium, Acharya, B. S., Agudo, I., et al

2019, Science with the Cherenkov Telescope Array, doi:10.1142/10986

Coerver, A., Wilcox, P., Zhang, H., et al. 2019, ApJ, 878, 126

Cordes, J. M., Freire, P. C. C., Lorimer, D. R., et al. 2006, ApJ, 637, 446

Crutcher, R. M. 2012, ARA\&A, 50, 29

Davidson, J. A., Werner, M. W., Wu, X., et al. 1992, ApJ, 387, 189

Dubus, G. 2013, A\&A Rev., 21, 64

Gelfand, J. D., Gaensler, B. M., Slane, P. O., et al. 2007, ApJ, 663, 468

Güver, T., \& Özel, F. 2009, MNRAS, 400, 2050

H.E.S.S. Collaboration, Abramowski, A., Aharonian, F., et al. 2016, Nature, 531,476

H.E.S.S. Collaboration, Abdalla, H., Abramowski, A., et al. 2018, A\&A, 612, A2

Jardin-Blicq, A., Marandon, V., \& Brun, F. 2019, in International Cosmic Ray Conference, Vol. 36, 36th International Cosmic Ray Conference (ICRC2019), 706

Lazzati, D., \& Stella, L. 1997, ApJ, 476, 267

Liu, L., \& Pang, X. 2019, The Astrophysical Journal Supplement Series, 245, 32

Lopez-Coto, R., Marandon, V., Brun, F., \& HESS collaborations. 2017, ArXiv e-prints, arXiv:1708.03137

MAGIC Collaboration, Acciari, V. A., Ansoldi, S., et al. 2019, arXiv e-prints, arXiv: 1911.06680

Martin, J., Torres, D. F., Cillis, A., \& de Oña Wilhelmi, E. 2014, MNRAS, 443, 138

Mori, K., Gotthelf, E. V., Hailey, C. J., \& Hord, B. J. 2017, ApJ, 848, 80

Mori, K., Hailey, C. J., Krivonos, R., et al. 2015, ApJ, 814, 94

Nice, D. J., Altiere, E., Bogdanov, S., et al. 2013, ApJ, 772, 50

Pandey, A., Gupta, A. C., \& Wiita, P. J. 2017, The Astrophysical Journal, 841,123

Reynolds, S. P., \& Chevalier, R. A. 1984, ApJ, 278, 630

Rho, C. D. 2017, ArXiv e-prints, arXiv:1708.03726

Rice, T. S., Goodman, A. A., Bergin, E. A., Beaumont, C., \& Dame, T. M. 2016, ApJ, 822, 52

Rieger, F. M., de Oña-Wilhelmi, E., \& Aharonian, F. A. 2013, Frontiers of Physics, 8, 714

Tavani, M., \& Arons, J. 1997, ApJ, 477, 439

The Fermi-LAT collaboration. 2019, arXiv e-prints, arXiv:1902.10045

van Velzen, S., Falcke, H., Schellart, P., Nierstenhöfer, N., \& Kampert, K.-H. 2012, A\&A, 544, A18

Wik, D. R., Hornstrup, A., Molendi, S., et al. 2014, ApJ, 792, 48

Xin, Y., Zeng, H., Liu, S., Fan, Y., \& Wei, D. 2019, ApJ, 885, 162

Zabalza, V. 2015, Proc. of International Cosmic Ray Conference 2015, 922 